\title{
Efficient Photodetector Based on Graphene Quantum Dots and Silicon Nanowires Junction
}

\author{
Iuliana Mihalache, Mihaela Kusko, Antonio Radoi \\ National Institute for Research and Development in Microtechnologies - IMT Bucharest \\ 077190, Bucharest, Romania \\ iuliana.mihalache@imt.ro; mihaela.kusko@imt.ro; antonio.radoi@imt.ro
}

\section{Extended Abstract}

Photodetectors based on silicon nanowires arrays target the spectral response from short-wavelength infrared to visible range and have attracted a grown interest due to their high sensitivity and photoresponse suitable for a wide variety of application. In this work, we demonstrate the fabrication and functionality of a core-shell heterojunction photodetector based on multilayers of graphene quantum dots $\left(\mathrm{GQD}^{\mathrm{PEI}} \mathrm{s}\right)$ and silicon nanowire arrays prepared by silver assisted chemical etching of p-type silicon wafer. Few nanometers quasi-spherical graphene quantum dots $\left(\mathrm{GQD}^{\mathrm{PEI}} \mathrm{s}\right)$ with appropriate strong yellow emission and 7\% quantum yield were synthesized through a bottom up approach from glucosamine and poly(ethyleneimine) and constitute the carbonaceous material enveloping the nanowire core, finally metal electrodes were deposited. GQDs unique properties [1,2] involved in light harvesting such as direct large optical bandgap sizable upon excitation and high absorption coefficients have been proven to facilitate the photocurrent conduction, leading to significant enhancement of device photodetection performance. The type I heterojunction band alignment with a large barrier potential created at quantum dots/ SiNWs interface was responsible for dark currents suppression observed in the current-voltage characteristics of $\mathrm{GQD}^{\mathrm{PEI}} \mathrm{s}$ based device. But, the remarkable values achieved for the ITO/nL-GQD ${ }^{\mathrm{PEI}} \mathrm{s} / \mathrm{p}$ SiNWs/Al photodetectors, such as high responsivity $\sim 25 \mathrm{~A} / \mathrm{W}$ and detectivity $\sim 9 \times 10^{12}$ Jones and EQE values that far exceed $100 \%$ at an applied bias of $4 \mathrm{~V}$, should be associated with complementary governing mechanisms. The experimental analysis indicated that the large gain achieved in $\mathrm{GQD}^{\mathrm{PEI}} \mathrm{S}$ based heterostructures arises from the interplay of various mechanisms mediated by $\mathrm{GQD}^{\mathrm{PEI}} \mathrm{S}$ : (i) trap induced carrier injection on nanowires leading to the generation of extra charges in device; (ii) improvement of the top contact, and consequently the collection of carriers; (iii) extension of light absorption due to the large band gap of $\mathrm{GQD}^{\mathrm{PEI}}$; (iv) photoelectric conversion mechanisms activated by $\mathrm{UV}$-vis absorption and down-conversion of light.

\section{References}

[1] X. Li, et al, "Carbon and graphene quantum dots for optoelectronic and energy devices: a review," Adv. Funct. Mater., vol. 25, no. 31, pp. 4929-4947, 2015.

[2] I. Mihalache, A. Radoi, M. Mihaila, C. Munteanu, A. Marin, M. Danila, M. Kusko, C. Kusko,"Charge and energy transfer interplay in hybrid sensitized solar cells mediated by graphene quantum dots," Electrochim. Acta, vol. 153, pp. 306-315, 2015. 